\title{
Optimization of Cúk Voltage Regulator Parameters for Better Performance and Better Efficiency
}

\author{
Walid Emar ${ }^{1}$ \\ Faculty of Engineering/Electrical \\ Engineering Department \\ Isra University \\ Amman, 11622 Jordan
}

\author{
Zakariya Al-omari ${ }^{2}$ \\ Faculty of Engineering/Renewable \\ Engineering Department \\ Isra University \\ Amman, 11622, Jordan
}

\author{
Omar A. Saraereh ${ }^{3}$ \\ Faculty of Engineering/Electrical \\ Engineering Department \\ The Hashemite University Zarqa \\ 13115, Jordan
}

\begin{abstract}
This paper discusses the harmonic distortion and voltage-current ripple minimization of a Cúk regulator based on the design optimization of its parameters using multichannel connection with uncoupled smoothing filters. The main attention is focused on the analysis and simulation of the fundamental and two-phase parallel connection of Cúk regulator with uncoupled smoothing filters. A detailed analysis has been done to show the benefits of uncoupled smoothing filters and their positive impact on balancing the energy compensation between the capacitors and inductors of the double-phase Cúk regulator as compared to conventional one. As a result of that the dc source current values do not go from positive to negative and vice versa as in the case of a fundamental connection which does not cause any saturation problem for the regulator. In general, multichannel parallel connection of Cúk regulators with uncoupled smoothing filters has ingrained benefits such as eminent current distribution characteristics, sacredness to component tolerance, reduction of parasitic effects and relief in current control complexity. Specifically, by employing double-phase connection with uncoupled smoothing filters for these regulators, overall current fluctuation can be effectively reduced by more than $25 \%$ after introducing the double-phase connection, compared to that of simple connection. Moreover, it is proved that the output voltage ripple of the double-phase connection is also reduced by more than $25 \%$ from that of the fundamental connection. Computer simulations using Simplorer 7 or Matlab and Excel have been done to validate the concepts.
\end{abstract}

Keywords-Cúk regulator; smoothing filters; double-phase connection; overall current ripple

\section{INTRODUCTION}

Due to environmental problems, the development of renewable energy has received much attention. Moreover, the green-house emissions of the generation system should be reduced according to international regulations [1]. One of the alternative energy resources is fuel cells that have attracted a great deal of attention.

To improve the performance of the fuel cell system, a fuel cell DC-DC converter with an appropriate controller is needed to regulate the energy flow and automatically adjust the output voltage of the converter [2]. The Dc-Dc chopper must be highly efficient and with less ripple.

A Cúk converter is a common type of converter which is using both buck and boost circuits converter will Change the voltage to a low or higher voltage level based on the characteristics that are designed for it [2].

Over the past few decades, the research of power electronics has focused on the development of multichannel parallel connections of DC-DC regulators in order to increase the ability of energy 2 processing and improve the reliability of the performance electronic system. The advantages of the regulator design using interlaced parallel regulators are the elimination of the ripple in both the input and output voltage and current curve waveforms to the maximum extent [3-5]. This will cause a lower value of the amplitude of the ripple and a high frequency in fluctuation in the resulting input and output waves. In addition, multichannel parallel power regulator connections reduce maintenance, increase reliability and tolerate faults. The insertion technique generally consists of shifting the control signals of several regulatory cells connected in parallel, and operating at the same switching frequency. The maximum benefits of interlacing can be achieved at a specific operating point. In general, for the Cúk regulator having one filter, the topology of one switch gives satisfactory performance in most applications, where the output voltage is greater than the input voltage but with a negative sign.

However, in a number of applications such as voltage correction circuits, energy saving and storage, and transformation of distributed power and solar systems, the performance of the Cúk regulator can be improved by implementing a Cúk with multiple channels in parallel and several smoothing filters.

The time-varying transfer function and parasitic effects of energy storage elements of a Cúk regulator leads to a positive/negative variation in the value of the source current and in a distortion in the output voltage and current. However, the current sourcing behavior of Cúk regulator helps for the use of parallel connection which can help to solve previously mentioned problems and can help paralleling many PV panels on the same bus of Common Coupling [4-7].

This paper proposes a new two-phase connection topology with uncoupled smoothing filters based on Cúk regulator with two-switch two-diode connected in parallel. Multichannel Cúk regulators operating in continuous mode of the filter current have better use of power equipments and devices, increasing power efficiency, lower losses and lower overall current 
fluctuations. However, in some cases, it is also acceptable that a regulator operating in a discontinuous current mode of the filter is required. For example, multi-channel DC regulators operating in a discontinuous current mode have a lower diode loss in the case of a reverse-recovery regime and a lower loss of switching on the transistor [6-9].

Moreover, the main problem of the design between parallel channels is the simultaneous distribution of currents into individual channels. It can be pointed out that if two similar but independently controlled Cúk regulators with the same input and output voltage, the regulator with 3 a larger duty cycle can operate in the continuous mode of the filter current, while the other will work in the discontinuous current mode of the filter. In these conditions, the regulator in the continuous current mode will take over any additional loading current. So, the current sharing is very sensitive to the mismatch in the duty cycle. The design of the control circuits for equal current sharing was discussed in some literature [9-12]. Conventional multi-channel Cúk regulator is typically used for applications with input current and voltage to output current and voltage conversion. An example is that the interlaced Cúk regulator is sometimes used in applications with high power to eliminate the losses of reverse - recovery of the rectifier via the control of Cúk regulators on the boundary of continuous or discontinuous current, so that the switches are switched on, When the current over the corresponding rectifier is zero. In addition, the Cúk regulator is also used in solar systems for the production of large DC voltages from PV modules.

\section{Simple CÚK REGULATOR}

The one-channel Cúk regulator is illustrated in "Fig. 1". The circuit model of such a regulator comprises a DC input voltage source, input filter $L_{1}$, controllable switch $S_{1}$, forward capacitor $C_{1}$, diode $D_{1}$, filter $L_{2}$, output filter capacitor $C_{o}$ and load resistance $R$. Its principle of operation explained in "Fig. 2" is currently known.

The Cúk voltage regulator provides an output voltage with a value smaller or larger than the input voltage value, but it is opposite to the input voltage. Its operation can be divided into two regimes: Regime 1 starts when the $S_{1}$ switch is on during the $t_{1}$ time, and its freewheeling diode is off. The current through filter $L_{1}$ raises linearly while simultaneously the $C_{1}$ capacitor voltage powering diode $D_{1}$ in the reverse direction which keeps it in the off time [17].

The $C_{1}$ capacitor is charged with energy through the circuit created by $C_{o}$, load and $L_{2}$. Regime 2 starts with the main switch $S_{2}$ in the off regime; the diode leads the currents of the $L_{1}$ and $L_{2}$ filters, while the $C_{1}$ capacitor is charged with a current of the $\mathrm{L}_{1}$ filter and the input power, $\mathrm{V}_{\mathrm{s}}$. The energy stored in the $L_{2}$ filter is now transferred to the load. The steady voltage and current curve waveforms for $1 / 2 \leq k \leq 1$ are shown in "Fig. 3".

A positive property of this connection is the continuous current at the input and output of the regulator. The disadvantages of the Cúk regulator represent a large number of reactive components and high current voltage stresses on the switch, diode and capacitor $\mathrm{C}_{1}$ and as a result of that the source current flowing through the DC source, $i_{s}$ may have negative values for a short time during $0 \leq k \leq 1 / 2$, as shown in "Fig. 4".

To obtain the DC voltage transfer function, we use the principle that the average current through the capacitor is zero for steady-state operation. Suppose the $L_{1}$ and $L_{2}$ filters are large enough to allow their current fluctuation to be decreased. The $\mathrm{C}_{1}$ capacitor is at steady state if

$I_{L 1} t_{1}=I_{S} t_{1}=I_{L 2} t_{2} \Rightarrow I_{S} D T=I_{L 2}(1-D) T$

For a lossless regulator, the input power is equal to the output power

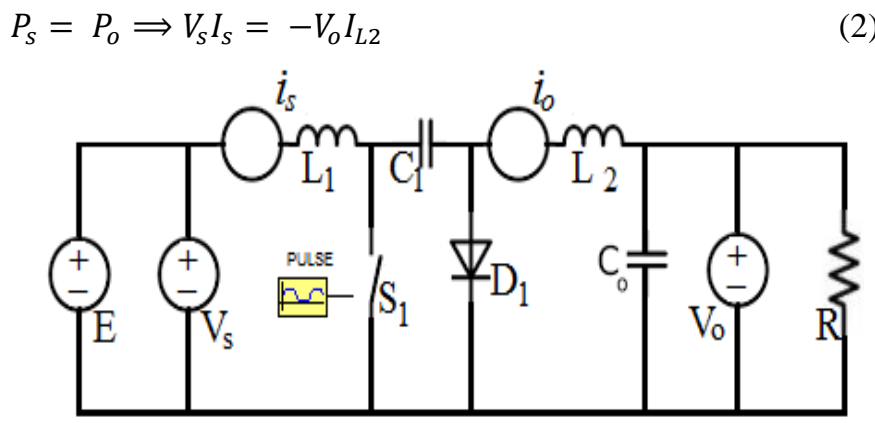

Fig. 1. Cúk Regulator Equivalent Circuit.

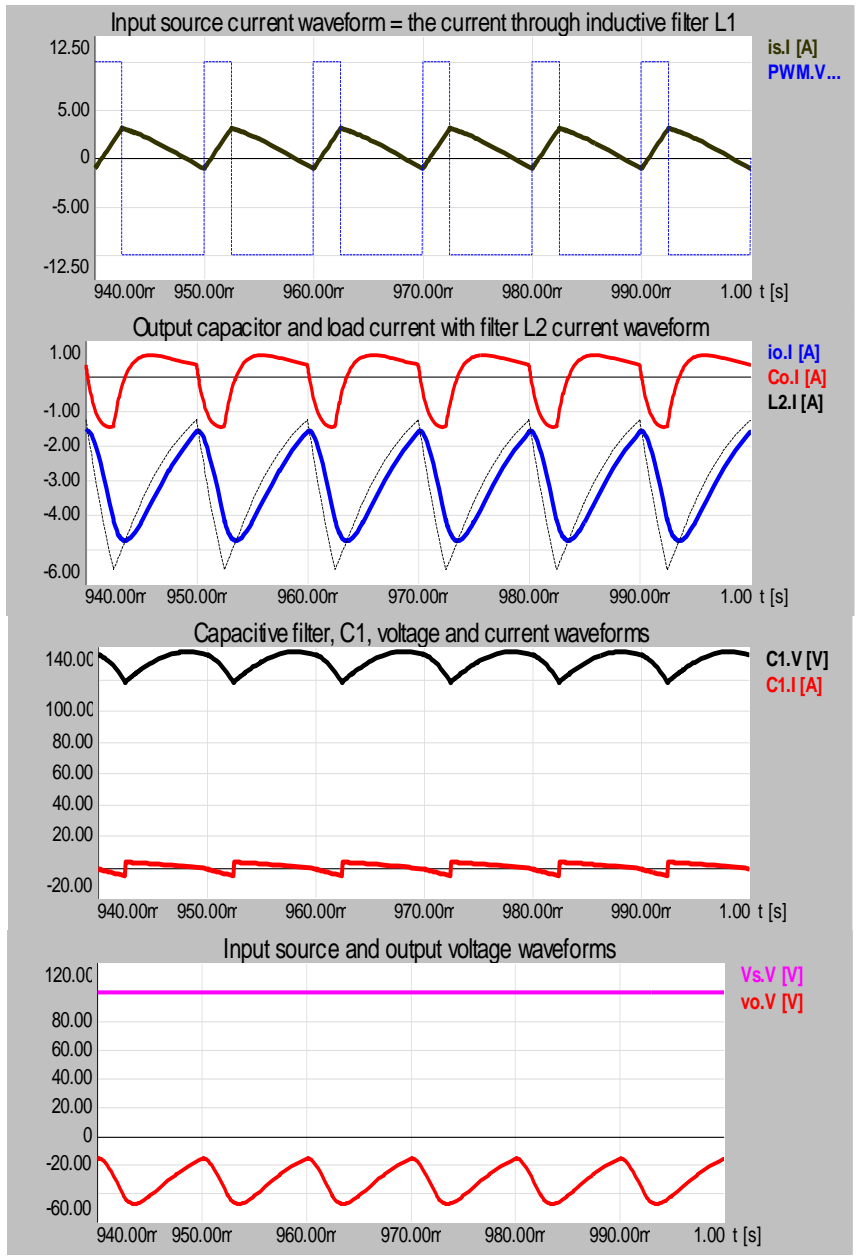

Fig. 2. Cúk Regulator with its Voltage and Current Waveforms for $0 \leq k \leq$ $1 / 2$. 


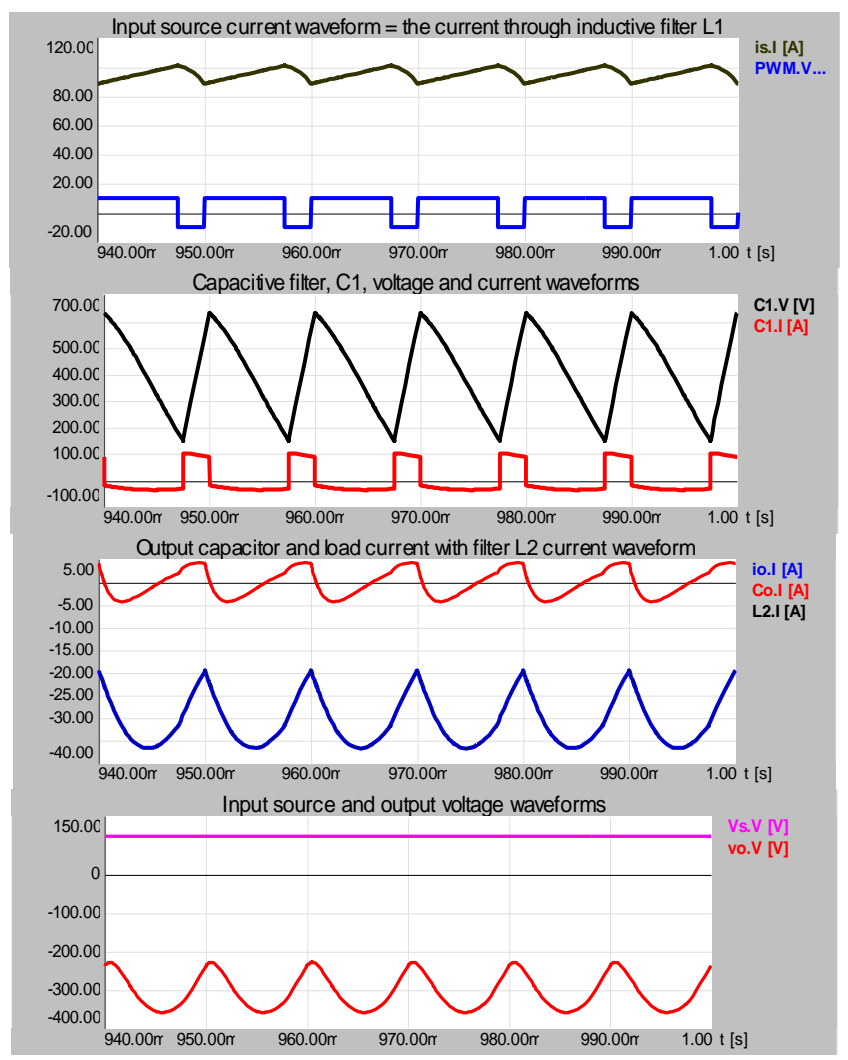

Fig. 3. Cúk Regulator with its Voltage and Current Waveforms for $1 / 2 \leq$ $k \leq 1$.

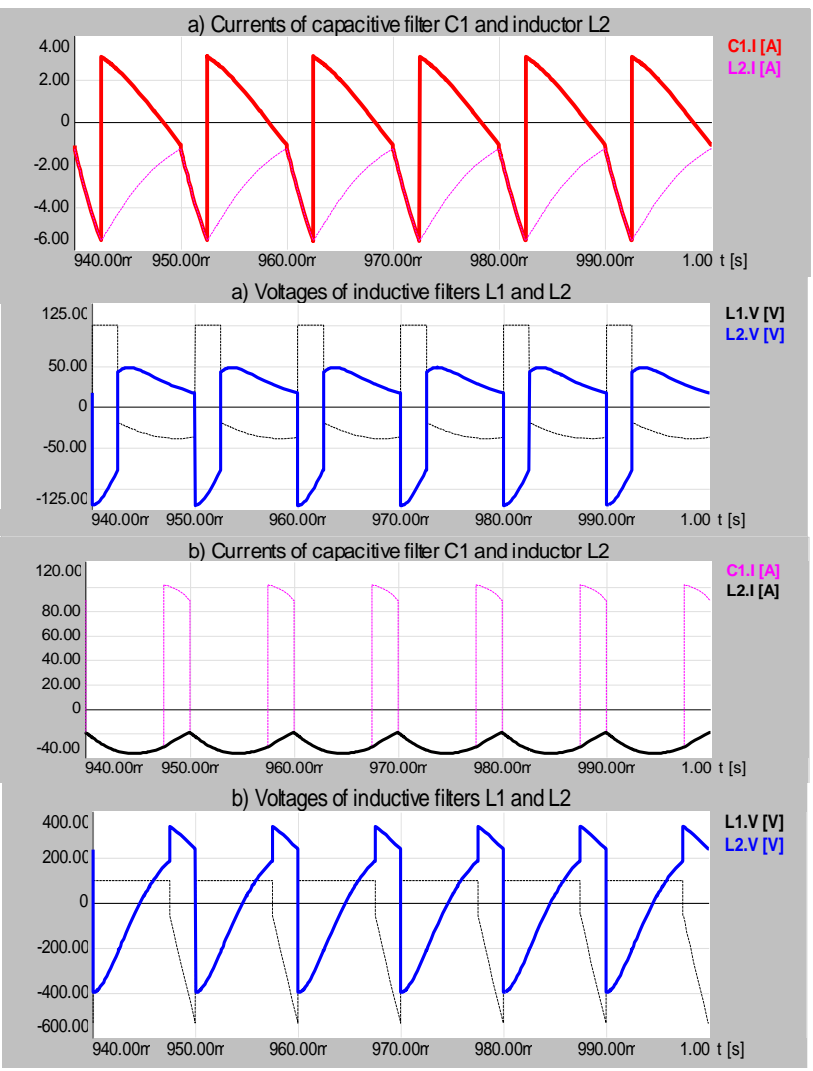

Fig. 4. Aditional Waveforms of Cúk Regulator for: a) $k \leq 1 / 2$ and b) $1 / 2 \leq k \leq 1$.
Combining these two equations, the dc voltage transfer function of the Cúk regulator is

$V_{o}=-\frac{k}{1-k} V_{s}$

When transistor $S_{1}$ is off, capacitor $C_{1}$ is charged by the input current for $t=t_{2}$. The average charging current of capacitor $C_{1}$ is $I_{C 1}=I_{S}$ and the peak-to-peak ripple voltage of the capacitor $\mathrm{C}_{1}$ is:

$\Delta V_{c 1}=\frac{1}{C_{1}} \int_{0}^{t_{2}} I_{c 1} d t=\frac{1}{C_{1}} \int_{0}^{t_{2}} \frac{\Delta i_{s}}{4} d t=\frac{\Delta i_{s} t_{2}}{4 C_{1}}$

Substituting for $t_{2}=\frac{1-k}{f}$ and for $\Delta i_{s}=\frac{\mathrm{kV}_{\mathrm{s}}}{f L}$, Eq. (4) becomes:

$\Delta V_{c 1}=\frac{\mathrm{V}_{\mathrm{s}}}{4 f^{2} L C_{1}} k(1-k)$

When the main switch is on, it carries currents of filters, $L_{1}$ and $L_{2}$, which results in a high peak current through this transistor. And because the capacitor provides the energy transfer, the fluctuation of the capacitor $C_{1}$ current is also very high. Therefore, the peak-to-peak ripple of the currents flowing into both inductors $i_{1}$ and $i_{2}$ for a closed or open switch is:

$\Delta i_{1}=\frac{k V_{S}}{f L}$

$\Delta i_{2}=\Delta i_{o}=\frac{V_{c 1}-V_{o}}{f L} k=\frac{-V_{o}}{f L}(1-k)=\frac{k V_{S}}{f L}$

Since the peak-to-peak ripple of the load current, $\Delta i_{o}$, is the same as the ripple of inductor current, $\Delta i_{2}$, we get for the peakto-peak ripple of voltage $\Delta \mathrm{V}_{\text {co }}$ :

$\Delta V_{c o}=\frac{1}{C_{o}} \int_{0}^{T / 2} I_{c o} d t=\frac{1}{C_{o}} \int_{0}^{T / 2} \frac{\Delta i_{2}}{4} d t=\frac{\Delta i_{2}}{8 f C_{o}}=\frac{k V_{S}}{8 f^{2} C_{o} L}$

\section{MUlTiChANNEl CÚK REGULATOR WITH UNCOUPLED FILTERS}

A circuit diagram for a Conventional two-channel connection of Cúk regulator with uncoupled filters $L_{1}, L_{2}$ is shown in "Fig. 5". Such regulator has two legs connected in parallel, $S_{1}, D_{1}$ and $S_{2}, D_{2}$. They are supplied from a dc source $\mathrm{V}_{\mathrm{S}}$ and they are switched on at different instants of time with a time displacement of $T / n$ ( $T$ is the operating period of each switch and $n$ is the number of channels of the regulator). The load comprises the following elements: resistor $\mathrm{R}$, uncoupled smoothing filters $L_{1}, L_{2}, L_{3}$ and $L_{4}$, which are inactive electric components created from a wire coil wound around an eliminated magnetic core to have their magnetic flux within all turns of the coil winding. The magnetic field generated from them is much stronger than the one generated by the single smoothing filter coil due to the induced voltage produced at their terminals as a result of the current flowing into both of them.

The current in filter $L_{1}$ is assumed to increase during its ontime, $i_{1}$ linearly from $I_{L a}$ to $I_{L b}$. During the off-time of switch $\mathrm{S} 1$ and on-time of $D_{1}$, the forward capacitor $C_{1}$ is discharging and therefore, current $i_{1}$ decreases linearly from $I_{L b}$ to $I_{L a}$, and:

$V_{s}-V_{c 1}=L \frac{\Delta i_{1}}{t_{2}} \Rightarrow t_{2}=L \frac{\Delta i_{1}}{V_{s}-V_{c 1}}$ 


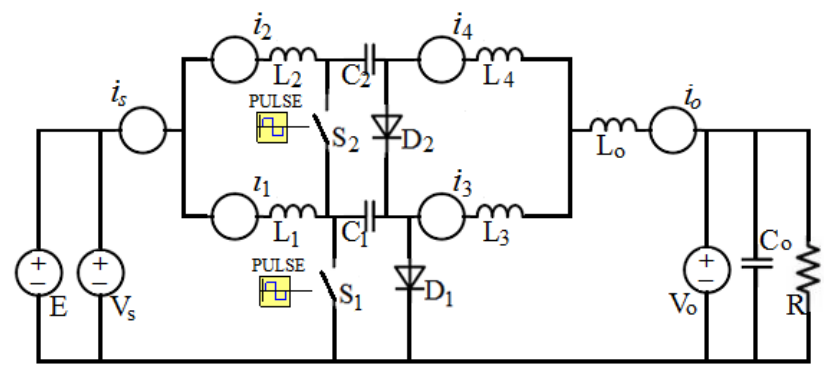

Fig. 5. Conventional Cúk Regulator with uncoupled Filters.

The peak-to-peak ripple of the phase current, $\Delta i_{1}$ under steady-state conditions should be same whether the switch is on or off, and it is obtained for the whole operating period of the regulator, $\boldsymbol{T}$, as shown in "Fig. 6".

Peak-to-peak ripple in regime of $1 / 2 \leq \mathrm{k} \leq 1$

Traditionally, the multichannel Cúk regulator having smoothing filters magnetically uncoupled perfectly decreases the overall voltage-current peak-to-peak ripples at the input and output of the regulator. It also significantly improves as said before the power efficiency as compared to the basic connection of Cúk regulator and helps improving the balance of energy transfer between energy storage elements. Therefore, the regulator never gets into a saturation problem and its dc input current never goes from positive to negative values. These investigations are recorded for typical and linear design conditions of the filters, whereas $L_{1}=L_{2}=L_{3}=L_{4}=L$. Therefore, the averaged values of currents into both channels are divided between filters, $L_{1}, L_{2}$ equally by regulating the duty cycle of every switch in each leg with a time displacement of $T / 2$ [17-19].

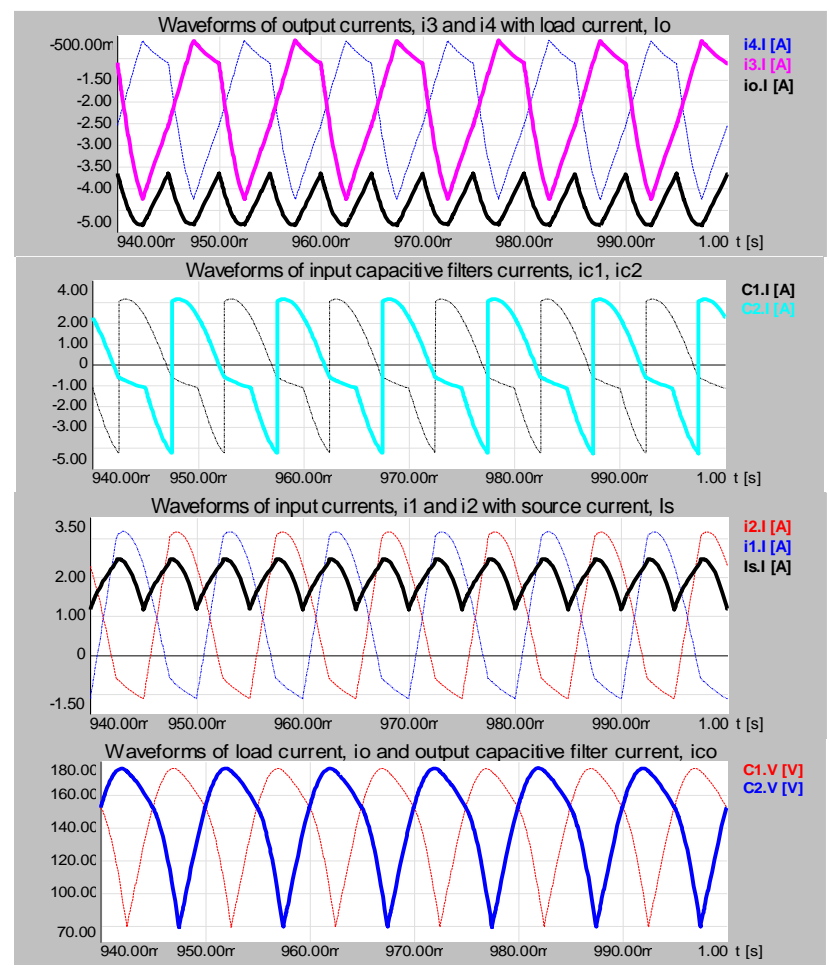

Fig. 6. Current and Volatge Steady state Waveforms of Two-Channel Connection of Cúk Regulator for $0 \leq \mathrm{k} \leq 1 / 2$.
Same currents but with opposite directions are flowing into filters, $L_{3}$ and $L_{4}$. So, considering a continuous operating mode of conduction, then the mathematical model representing the system when $S_{1}$ is switched on, for a time $t_{1}$ is as follows:

$V_{s}=L \frac{I_{L a}-I_{L b}}{t_{1}} \Rightarrow t_{1}=L \frac{\Delta i_{1}}{V_{S}} \Rightarrow \Delta i_{1}=\frac{k V_{S}}{f L}$

The current in filter $L_{1}$ is assumed to increase during its on-time, $t_{1}$ linearly from $I_{L a}$ to $I_{L b}$. During the off-time of switch $S_{1}$ and on-time of $D_{1}$, the forward capacitor $C_{1}$ is discharging and therefore, current $i_{1}$ decreases linearly from $I_{L b}$ to $I_{L a}$, and:

$V_{s}-V_{c 1}=L \frac{\Delta i_{1}}{t_{2}} \Rightarrow t_{2}=L \frac{\Delta i_{1}}{V_{s}-V_{c 1}}$

The peak-to-peak ripple of the phase current, $\Delta i_{1}$ under steady state conditions should be same whether the switch is on or off, and it is obtained for the whole operating period of the regulator, $T$, as follows:

$T=t_{1}+t_{2}=L \frac{\Delta i_{1}}{V_{S}}+L \frac{\Delta i_{1}}{V_{S}-V_{c 1}} \Rightarrow \Delta i_{1}=\frac{V_{S}\left(V_{S}-V_{c 1}\right)}{f L\left(2 V_{S}-V_{c 1}\right)}$

The averaged voltage across capacitor $C_{1}, V_{C_{1}}$ is obtained from the equality of the fluctuation in the on and off-time as follows:

$\Delta i_{1}=\frac{\mathrm{V}_{\mathrm{s}}}{L} t_{1}=\frac{\mathrm{V}_{\mathrm{s}}}{L} k T=\frac{V_{s}-V_{c 1}}{L} t_{2}=\frac{V_{s}-V_{c 1}}{L}(1-k) T$

$\Rightarrow V_{c 1}=\frac{(1-2 \mathrm{k})}{(1-\mathrm{k})} \mathrm{V}_{\mathrm{s}}$

Similar thoughts may be applied for the second channel current, $i_{2}$. Therefore, because the smoothing filters $\boldsymbol{L}_{\mathbf{1}}=\boldsymbol{L}_{\mathbf{2}}=$ $\boldsymbol{L}$ are identical, the peak-to-peak ripple of the regulator input current $i_{s}$ may be determined for $\mathbf{0} \leq \boldsymbol{k} \leq \mathbf{1} / \mathbf{2}$ as shown in "Fig. 7", when one main switch is on and the other is off as follows:

$\Delta i_{s}=\frac{V_{s}}{L} t_{1}-\frac{V_{s}-V_{c 1}}{L} t_{1} \Rightarrow \Delta i_{s}=\frac{V_{c 1}}{f L} k=\frac{k(1-2 k)}{(1-k) f L} V_{s}$

Concerning $\Delta i_{s}$ in the region of $1 / 2 \leq k \leq 1$ as shown in "Fig. 7" when both switches are on, it may be obtained as follows:

$\Delta i_{s}=2 \frac{V_{s}}{L}\left(t_{1}-\frac{T}{2}\right) \Rightarrow \Delta i_{s}=\frac{(2 k-1)}{f L} V_{s}$

Concerning the output currents, $i_{3}$ and $i_{4}$ through filters, $L_{3}$ and $L_{4}$, let's assume that current $i_{4}$ increases linearly in time $i_{1}$ :

$V_{c 1}-V_{o}=L_{4} \frac{\Delta i_{4}}{t_{1}} \Rightarrow t_{1}=L \frac{\Delta i_{4}}{V_{c 1}-V_{o}}$

And in time $t_{2}$ :

$V_{o}=-L_{4} \frac{\Delta i_{4}}{t_{2}} \Rightarrow t_{2}=-L \frac{\Delta i_{4}}{V_{o}}$

Combining 14 and 15:

$T=t_{1}+t_{2} \Rightarrow \Delta i_{4}=\frac{V_{o}\left(V_{c 1}-V_{o}\right)}{f L\left(2 V_{o}-V_{c 1}\right)}=\frac{V_{s}}{f L} k$

Equating the input power to the output power for a lossless regulator and Eq. (16) to Eq. (17), we get for the output voltage and current: 
$V_{o}=-\frac{k V_{s}}{1-k}, \quad I_{o}=\frac{1-k}{k} I_{s}$

The fluctuation of load current $i_{o}$ may be determined for $\mathbf{0} \leq \boldsymbol{k} \leq \mathbf{1 / 2}$ when one main switch is on and the other is off as:

$\Delta i_{o}=\frac{V_{c 1}-V_{o}}{L} t_{1}+\frac{V_{o}}{L} t_{1} \Rightarrow \Delta i_{o}=\frac{V_{c 1}}{f L} k=\frac{(1-2 k) k V_{s}}{(1-k) f L}$

When both switches are on, $\Delta \boldsymbol{i}_{\boldsymbol{o}} \Delta i_{\mathrm{o}}$ may be obtained for $1 / 2>k>1$ as:

$\Delta i_{o}=2 \frac{V_{c 1}-V_{o}}{L}\left(t_{1}-\frac{T}{2}\right) \Rightarrow \Delta i_{o}=\frac{(2 k-1)}{f L}\left(V_{c 1}-V_{o}\right)=$ $\frac{(2 k-1)}{f L} V_{s}$

The average value of capacitor $\mathrm{C}_{\mathrm{o}}$ current, which flows for $\boldsymbol{T} / \mathbf{2}$, is $\boldsymbol{I}_{\boldsymbol{c o}}=\Delta \boldsymbol{i}_{\boldsymbol{o}} / \mathbf{4}$ and therefore, the peak-to-peak ripple voltage of capacitor $\mathrm{C}_{\mathrm{o}}$ is:

$\Delta v_{c o}=\frac{1}{C_{o}} \int_{0}^{\frac{T}{2}} I_{c o} d t=\frac{1}{C_{o}} \int_{0}^{\frac{T}{2}} \frac{\Delta i_{o}}{4} d t=\frac{\Delta i_{o}}{8 f C_{o}}$

Concerning the fluctuation of the voltage on capacitors, $\boldsymbol{C}_{\mathbf{1}}$, or $\boldsymbol{C}_{2}$ is:

$$
\begin{gathered}
\Delta v_{c 1}=\frac{1}{C_{1}} \int_{0}^{t_{2}} I_{c 1} d t= \\
\frac{1}{C_{1}} \int_{0}^{t_{2}} \frac{\Delta i_{1}}{4} d t=\frac{\Delta i_{1} t_{2}}{4 C_{1}}=\frac{\Delta i_{1}}{4 f C_{1}}(1-k)
\end{gathered}
$$

The peak-to-peak ripple, in this case, has the same value as that for the fundamental connection estimated in Eq. (5).

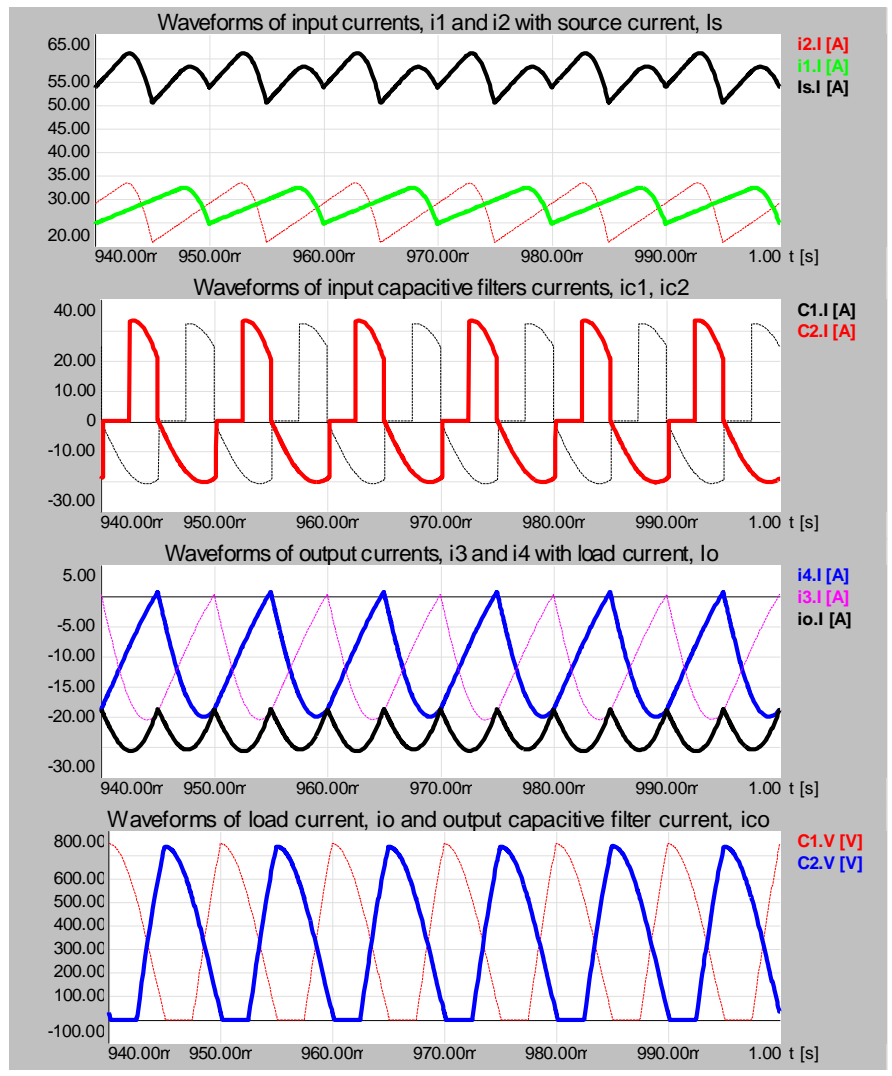

Fig. 7. Cúk Regulator with its Voltage and Current Waveforms for $1 / 2 \leq$ $k \leq 1$.
The current and voltage fluctuations are undesired behavior which should be improved since they adversely affect the losses and heating effects in the system load above the acceptable limits and consequently decreases the expected life of the battery in which the energy created by PV systems is stored. It also makes the regulation of the regulator worse since it generates a distortion not only in the current and voltage waves at the output of the regulator but also at its input which requires further control processors and makes its control more sophisticated and the dynamic behavior of the PV supply system unstable and less efficient [13-16].

\section{Simulation RESUlTs}

The peak-to-peak ripple of source and load current of the Cúk regulator with fundamental connection and double-phase connection with smoothing chokes is illustrated using previously derived equations as a function of duty time ratio, $k$, in "Fig. 8". The regulator simulation parameters for the current continuous regime are listed in "Table I" as [20-22].

The ripple of currents, $\boldsymbol{i}_{\boldsymbol{s}}$ and $\boldsymbol{i}_{\boldsymbol{o}}$ is directly proportional to the supply voltage $V_{s}$ and inversely proportional to the chopping frequency of the regulator $f$ and the filter inductance value $L$.

From "Fig. 8" and "Fig. 9" it is clear that the peak-to-peak source and load current ripple of double-phase connection of Cúk regulator with smoothing filters has smaller values than that for its fundamental connection. It should be mentioned that the number of smoothing filters for $\mathrm{n}$-channel connections is $\mathrm{n}$ filters, but compared to the basic connection, each filter is designed to the value of $1 / n$ of the source current.

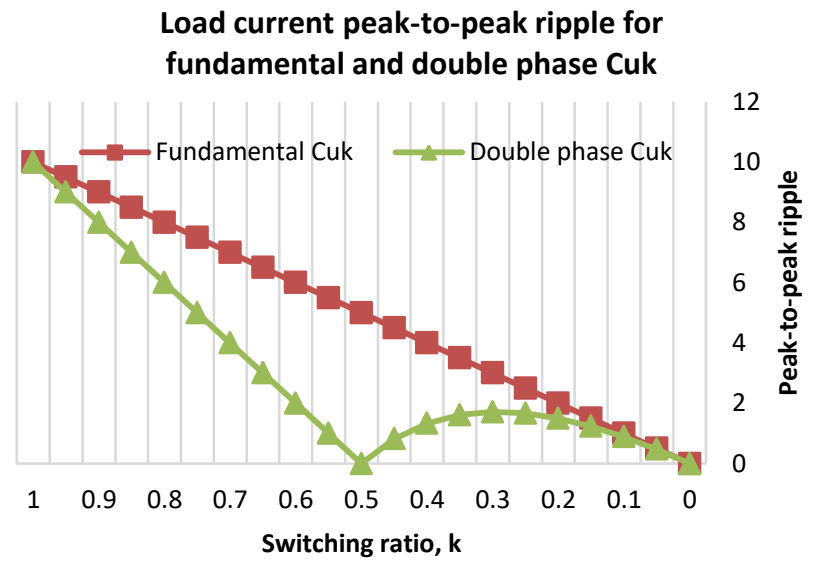

Fig. 8. Peak-to-Peak Ripple of Load Current $\Delta i o$ Versus Duty Ratio $k$.

TABLE. I. TeChNicAl PARAmEters of THE Simulated REGUlatoR

\begin{tabular}{|l|l|c|}
\hline Parameter & Symbol & Real value \\
\hline Smoothing filters: & $L_{l}=L_{2}=L_{3}=L_{4}$ & $10 \mathrm{mH}$ \\
\hline Regulator operating period: & $T$ & $10 \mathrm{~ms}$ \\
\hline Supply voltage & $V_{s}$ & $100 \mathrm{~V}$ \\
\hline Load resistance: & $R$ & $10 \Omega$ \\
\hline Input capacitive filter & $C_{l}=C_{2}$ & $100 \mu \mathrm{F}$ \\
\hline Output capacitive filter & $C_{o}$ & $100 \mu \mathrm{F}$ \\
\hline
\end{tabular}




\section{Peak-to-peak source current ripple versus switching ratio, $\mathrm{k}$ for double phase and basic Cuk}

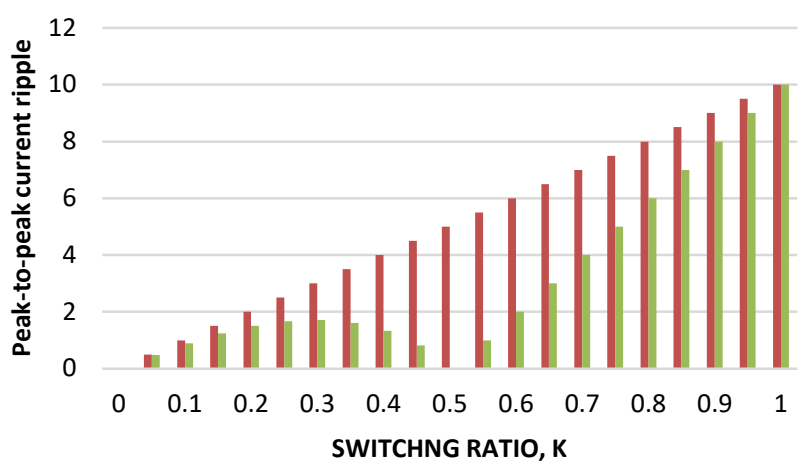

- Phase current ripple

Source current ripple of a two-phase connection

Fig. 9. Peak-to-Peak Ripple of Source $\Delta$ isversus Duty Ratio k.

In addition, "Fig. 10" shows that with an increase in switching frequency, current fluctuations in the two- channel connection are dramatically reduced compared to the underlying fundamental connection. The advantages of reducing fluctuations in the current waves due to multichannel connection of Cúk regulator result in the use of smaller filters and capacitors on the output and input of the regulator for the same requirements for the total peak-to-peak current and voltage ripple. Even the transient ripple of output voltage on the $C_{1}$ and $C_{2}$ channel capacitors can also be reduced due to smaller output coils, resulting in the use of smaller output capacitors. While the ripple on the channel capacitive filters, $C_{1}$ and $C_{2}$ does not change as shown in "Fig. 11" in blue, it is dramatically decreased on the output capacitive filter, $C_{o}$ for double-phase connection (in green) as compared to that of the fundamental connection in red [9, 21-24].

Peak-to-peak current ripple versus frequency

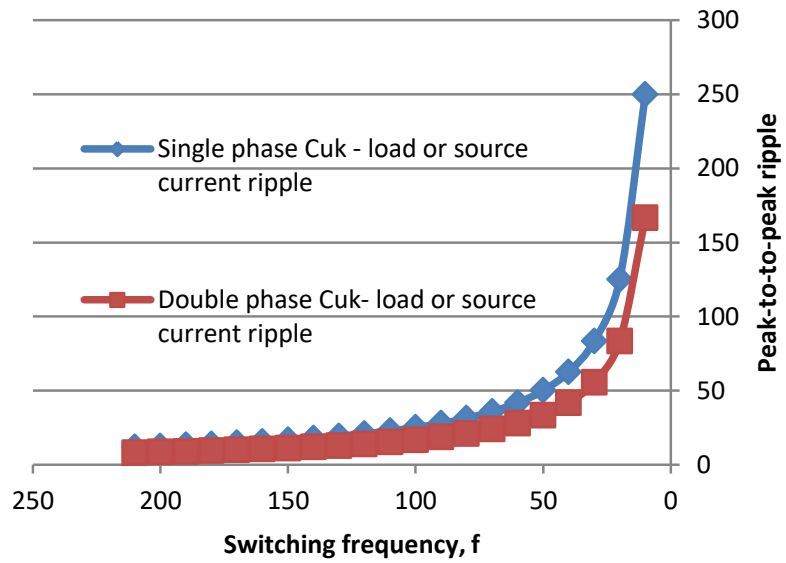

Fig. 10. Peak-to-Peak Ripple of Input forward and Output Capacitive Filter Voltages Versus Switching Ratio k.

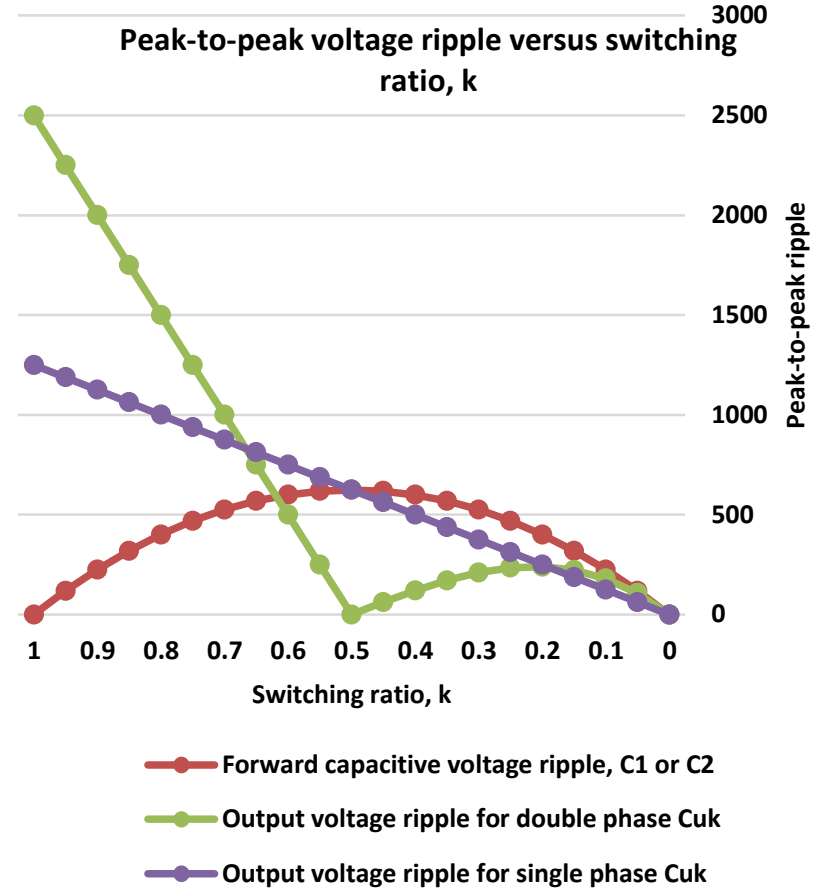

Fig. 11. Peak-to-Peak Ripple of Input forward and Output Capacitive Filter Voltages Versus Switching Ratio kfor Both Topologies of Cúk.

\section{CONCLUSION}

This paper focuses on the concept of total reduction of current and voltage ripple of the Cúk regulator using multichannel parallel connection with disconnected smoothing filters. A detailed analysis was performed, while simulations and experimental results were performed to verify the concept. In addition, it was found that the fluctuations in the channel currents will also be reduced compared to the basic connection of the regulator. This can increase efficiency and improve the system's dynamic performance. Furthermore, in the case of a single-phase Cúk regulator, the switching ratio should be carefully selected to achieve better results and to avoid parasitic effects of the capacitive and inductive filters. This is not a problem of the double-phase Cúk regulator with smoothing inductive filters that help to solve the parasitic effect of capacitive filters on the planar inductive filters.

- The simulations and experimental results obtained in this article lead to the following conclusion: Singlephase Cúk regulator may be given a priority when it is used for low power applications, for example, in telecom power supplies, smart home grid systems, and computer motherboards and power supplies where the parallel connection is not required.

- Two-phase connection with magnetically uncoupled/coupled filters is more favorable with respect to the present trend of high switching frequency, $f$. and better dynamic performance power electronic devices. These features of Cúk regulator results in a decrease of the demands to the material of smoothing inductive and capacitive filters used with it. 


\section{ACKNOWLEDGMENT}

Authors acknowledge the Presidency of Isra University that supports this research study and to any person, institution or department that supported any part of the study.

\section{REFERENCES}

[1] Walid Emar, Omar A. Saraereh: Analytical and Comparative Study of Different Types of Two-Leg Chopping up Regulator, (IJACSA) International Journal of Advanced Computer Science and Applications, Vol. 10, No. 5, 2019.

[2] Zakaria Al-Omari, A. Hamzeh, Sadeq A. Hamed, A. Sandouk, G. Aldahim, "A Mathematical Model for Minimizing Add-On Operational Cost in Electrical Power Systems Using Design of Experiments Approach", International Journal of Electrical and Computer Engineering (IJECE) Vol. 5, No. 5, October 2015, pp. 948 956.

[3] M. A. Z. A. Rashid, A. Ponniran, M. K. R. Noor, J. N. Jumadril, M. H. Yatim, A.N. Kasiran, "Optimization of PFC cuk converter parameters design for minimization of THD and voltage ripple”, International Journal of Power Electronics and Drive System (IJPEDS) Vol. 10, No. 1, March 2019, pp. 514-521.

[4] Walid Emar, Omar A. Saraereh, Karim Aljebory: Three Phase Three Level Voltage Fed Inverter for a Doubly Fed Induction Motor, Transylvanian Review, Transylvanian Review: Vol XXV, No. 22, November 2017.

[5] Walid Emar: Anaslysis, Modelling and Simulation of step up Converter using Matlab-simulink and simplorer, International Journal of modeling, simulation and scientific computing, (IJMSSC) Vol. 7, Issue 3.

[6] Zoubir Belgroun, Mustapha Hatti, Salah Hanani, "Power interface efficiency evaluation for photovoltaic system used in hydrogen production", Przegląd Elektrotechniczny, ISSN 0033-2097, R. 95 NR $6 / 2019$.

[7] M. Bildirici A. Karaarslan, "Analysis of Cuk Converter using PI and OCC Control Method", 13th International Conference on "Technical and Physical Problems of Electrical Engineering” 21-23 September 2017 Yuzuncu Yil University Van, Turkey.

[8] M. K. R. Noor, A. Ponniran, M. A. Z. A. Rashid, J. N. Jumadril, A. A. Bakar1, K. S. Muhammad, M. H. Yatim and A. N. Kasiran, "Optimization Parameter Design of SEPIC-Cuk Converter", International journal of Integrated Engineering Vol. 11, No. 1 (2019) 2732.

[9] Walid Emar, Aqel Musbah, and I. M. M. El Emary: "Two-channel connection of switching Converters", European Journal of Scientific Research, Vol. 16, No. 1, January 2007.

[10] A. Bin Ponniran et al., "Fundamental Operation of Marx Topology for High Boost Ratio DC-DC Converter,” IEEJ J. Ind. Appl., vol. 5, no. 4, pp. 329-338, 2016.

[11] A. N. K. and A. P. and M. A. H. and H. H. Hamzah, "A Study of 4level DC-DC Boost Inverter with Passive Component Reduction Consideration,” J. Phys. Conf. Ser., vol. 995, no. 1, p. 12062, 2018.

[12] A. Shahul, P. A. P. Oommen, and P. B. Cherian, "Self Lifted SEPICCuk Combination Converter," vol. 3, no. 2, pp. 36-43, 2016.

[13] J. Marjani, A. Imani, A. Hekmati, and E. Afjei, "A new dual output DCDC converter based on SEPIC and Cuk converters," in 2016 International Symposium on Power Electronics, Electrical Drives, Automation and Motion (SPEEDAM), 2016, pp. 946-950.

[14] A. Shahul, P. A. P. Oommen, and P. B. Cherian, "Self Lifted SEPICCuk Combination Converter," vol. 3, no. 2, pp. 36-43, 2016.

[15] A. N. K. and A. P. and M. A. H. and H. H. Hamzah, "A Study of 4-level DC-DC Boost Inverter with Passive Component Reduction Consideration,” J. Phys. Conf. Ser., vol. 995, no. 1, p. 12062, 2018.

[16] M. H. Yatim et al., "Symmetrical and asymmetrical multilevel inverter structures with reduced number of switching devices," Indonesian Journal of Electrical Engineering and Computer Science, vol. 11, no. 1, pp. 144-151, 2018.
[17] Muhammad H. Rashid. Power Electronics, Circuits, devices, and Applications, Electrical and Computer Engineering, University of West Florida, Pearson Education International, third edition, 2004.

[18] M. Jang, M. Ciobotaru, and V.G. Agelidis, "A One-Channel Grid Connected Fuel Cell System Based on a Boost-Inverter," IEEE Trans. Power Electronics. Appl., vol. 28, no. 1, pp. 279-289, Jan. 2013.

[19] T. D. Rachmildha, Y. Haroen, A. Muqorobin, and E. Rijanto "One Channel Boost Inverter Using Hybrid Modelling Approach," IEEE Conference on Power Engineering and Renewable Energy 2012, pp. 16, Jul. 2012.

[20] Rosas-CaroJ, Mayo-Maldonado J. DC-DC multiplier Cúk converter with resonant switching, Electric Power, ELSEVIER, Systems ResearchVolume 119, February 2015; Pages 83-90.

[21] Chierchie, F. Paolini, E.E. Discrete-time modeling and control of a synchronous buck converter .Argentine School of MicroNanoelectronics, Technology and Applications, 2009. EAMTA 2009.12 October 2009, pp. 5 - 10. ISBN 978-1-4244-4835-7.

[22] Su, J.H.; Chen, J.J.; Wu, D.S.; "Learning feedback controller design of switching converters via Matlab/Simulink" Education, IEEE Transactions on, Volume: 45, Issue: 4, Nov. 2002 Page(s): 307 -315.

[23] N.Mohan, T. M. Undeland, and W. P. Robbins, "Power Electronics, Converters, Applications, and Design,” John Wiley \& Sons, 2003.

[24] KhadmunW, Subsingha W. High Voltage Gain Interleaved DC Cúk Converter Application for Photovoltaic Generation System, Energy Procedia, ELSEVIER, 10th Eco-Energy and Materials Science and Engineering Symposium, Volume 34, 2013; Pages 390-398.

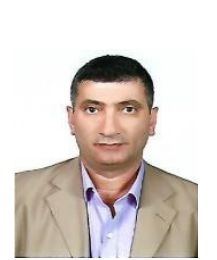

\section{AUTHORS' PROFILE}

Walid Emar received his B.Sc. and his M.Sc. degree in power electronics in 1996/1997 and his Ph.D. degree in power electronics and control in 2002 from the University of West Bohemia, Czech Republic. He worked for different institutes in his field in Czech rep. as Škoda Company, Japanese air-conditioning Daikin and Fuji Koyo. Currently, $\mathrm{He}$ is at Isra University, Jordan as a fulltime associate professor for teaching Energy Management for Master's degree students and control systems, electrical machines, in addition to power electronics and other subjects for undergraduate students. He is also engaged in research in control of power electronics and machinery control. He is also acting as a reviewer for Scopus and ISI Clarivate journals just like IJMSSC, INDERSCIENCE, ACTAPRESS.

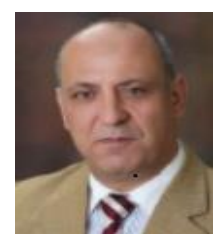

Zakaria Al-Omari, was born in Irbid Jordan on June 3, 1966. He obtained his MSc degree (1991), in Electrical Engineering/Power from the Faculty of Electrical Engineering, Vinnytsia State Polytechnic Institute, Ukraine and his $\mathrm{PhD}$ degree from the Faculty of Electrical Engineering, Vinnitsa National Technical University, Ukraine in 1998. Currently he is an Associate Professor at Renewable Engineering Department at Faculty of Engineering, Isra University in Amman, Jordan. His main interests are minimizing of power system losses, renewable energy, Power Electronics, load forecasting, reliability and efficiency. He has published 15 technical papers in Journals and international conferences.

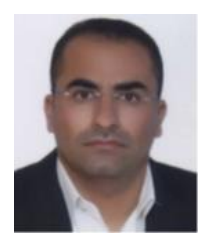

Omar A. Saraereh initially qualified as a Telecommunication Engineer 1999 from Mu'TAH University, Jordan; he then obtained a Master of Science Degree in Digital Communication Systems from England. In 2005 he completed his PhD in Electrical and Electronic Engineering/Mobile Communications from Loughborough University, England. Dr. Saraereh has Over 12 years of academic and practical experience in Electrical Engineering, Mobile Communications, Various Antennas Design, Fabrication \& Measurements, Radiation Hazards and Health Effects, and Wireless Communications. Dr. Saraereh has published many papers in various international journals and conferences. Currently Dr. Saraereh is an associate professor in the Department of Electrical Engineering at the Hashemite University/Jordan. 